Voix et Images

voixetimages

\title{
Borduas, peintre et... écrivain?
}

\section{Norman Pagé}

Volume 13, numéro 3 (39), printemps 1988

Jack Kerouac et l’imaginaire québécois

URI : https://id.erudit.org/iderudit/200736ar

DOI : https://doi.org/10.7202/200736ar

Aller au sommaire du numéro

\section{Éditeur(s)}

Université du Québec à Montréal

\section{ISSN}

0318-9201 (imprimé)

1705-933X (numérique)

Découvrir la revue

\section{Citer cet article}

Pagé, N. (1988). Borduas, peintre et... écrivain? Voix et Images, 13(3), 478-483.

https://doi.org/10.7202/200736ar d'utilisation que vous pouvez consulter en ligne.

https://apropos.erudit.org/fr/usagers/politique-dutilisation/ 


\section{Borduas, peintre et... écrivain?}

\section{par Norman Pagé, Université d'Ottawa}

À l'heure où le Musée d'art moderne de Paris présente une remarquable visite guidée du Minotaure d'André Breton, alors que le Musée des beaux-arts de Montréal expose une rétrospective des toiles de Borduas et le Musée du Québec, un accrochage d'œuvres des onze récipiendaires du prix Paul-Émile Borduas, voici que les Presses de l'Université de Montréal, lancent Écrits I de Paul-Émile Borduas 1 , cinquième volume de la collection«Bibliothèque du Nouveau Monde». Désormais, les écrits célèbres ou inconnus de Borduas voisineront - à la portée des intéressés - avec les tableaux du peintre révolutionnaire. Noir sur blanc.

Le mythe Borduas n'est pas d'hier, chacun le sait. Qu'en est-il, plus rigoureusement toutefois, de l'ensemble des écrits, publiés et inédits, de ce même Borduas? André-G. Bourassa, Jean Fisette et Gilles Lapointe nous l'apprennent. Ces trois chercheurs de l'Université du Québec à Montréal, en collaboration avec une forte équipe de consultants, assistants de recherche et autres adjoints, ont entrepris, non sans crainte (p. 11), de nous livrer une édition de la presque totalité de ses écrits et de leur reconnaître un statut littéraire (p. 13). Et qui plus est, de présenter ces textes en une édition critique qui, fondée sur l'établissement du texte original et sur la vérification de ses sources comme de sa réception, devrait contribuer d remplacer la connaissance mythique par la connaissance scientifique (p. 15).

Ces valeureux chercheurs livrent donc aujourd'hui, quelque dix ans après la mise en marche de ce projet, le résultat de leur analyse critique de milliers de pages manuscrites dans un imposant volume de 700 pages. L'édition de la correspondance et autres documents personnels de Borduas suivra dans un second volume, Écrits II.

Les textes compulsés, publiés ou inédits, dans Écrits I s'étendent sur une période qui court de 1933 à 1958. Aux fins de leur analyse critique respective, ils ont été répartis chronologiquement en cinq regroupements, lesquels constituent les cinq «parties» du volume. André-G. Bourassa s'est réservé la première (91 p.), 
la deuxième $(97$ p.) et la cinquième partie ( 65 p.) pour y traiter bon nombre d'œuvres moins connues où inédites de Borduas2. Jean Fisette signe la troisième partie (127 p.), celle qui contient Refus global, et Gilles Lapointe, la quatrième partie (110 p.) consacrée exclusivement à Projections libérantes. Les dix appendices qui suivent présentent, en 128 pages, des textes considérés de moindre importance, mais néanmoins nécessaires ou utiles aux spécialistes. La méthode d'analyse et la présentation d'ensemble de la matière est sensiblement la même pour chacune des parties đủ volume: introduction générale, établissement des textes, texte de base, annotations. Une excellente chronologie, mise à jour par Gilles Lapointe en renant compte de données inédites tirées de la correspondance et du journal intime de Borduas complète le tout, mais renvoie cependant au Paul-Émile Borduas de François-Marc Gagnon pour le répertoire complet des expositions auxquelles Borduas a participé de 1927 à $1960^{3}$.

La lecture des Ḱ des plus fervents admirateurs de Borduas. Qu'on en juge par cet aperçu des divers textes présentés et annotés.

Dans la première partie du volume, A.-G. Bourassa se limite aux quelques premiers écrits de Borduas datés d'avant 1940 et inédits jusqu'à ce jour. Le plus ancien de ces documents, Perspective, est un texte dactylographié de 48 pages, sans date, mais qui aurait vraisemblablement été rédigé et illustré en 1933, alors que Borduas était professeur de dessin dans diverses écoles du centre de Montréal et ce, à partir principalement des notes de cours prises a l'École des beaux-arts de Montréal; un cours, donc, assez élémentaire en lui-même et on ne peut plus classique, mais qu'il convient de révéler aux détracieurs de l'automatisme qui seraient étonnés de constater sur quelles bases classiques reposent les recherches spatiales des années quaronte (p. 55). Le deuxième écrit, Composition décorative, est un manuscric autographe de 44 pages, rédigé en 1935 et destiné aux étudiants de l'École du meuble. Borduas y aborde, avec le plus grand conformisme académique, diverses notions concernant l'art décoratif, notamment: la couleur, lharmonie des tons, la mise en mouvement et la composition, le tout dans l'optique particulière d'une école du meuble. Les troisième et quatrième écrits ne sont en somme que des procès-verbaux de deux Enquêtes sur l'art et l'artisanat du Québec effectuées par J.-M. Gauvreau, l'abbé A. Tessier et Borduas au cours des étés 1938 et 1939 prour le compte du ministère du Commerce et de lIndustrie du Québec. Borduas m'est que cosignataire d'une série de ces rapports 4 .

En deuxième partie du volume, Bourassa nous présente les publications de Borduas parues entre 1941 et 1943, soir aus cours des années où le peintre expose ses premières auvres et s'implique personmellement dans les diverses polémiques qui entourent le célèbre Salon des Indépendants. Du premier de ces textes, Réponse des Indépendarits Maillard (1941), Borduas n'est encore que cosignataire, il est vrai (ce qui explique que ce manifeste soit reporté en appendice), mais à coup sâr chacun l'y reconnaît comme l'un des tenants les plus convaincus de cette rupture qui a fait scandale. Le très bref article qui suit, Fusain, est un 
hommage d'occasion à un ami, Jacques G. de Tonnancour, celui, écrit Borduas qui sut très tôt laisser tomber les sottes formules [...] et nous introduire au paradis bien gardé de la beauté plastique (p. 165-166). Le demier texte de cette période, Manières de goûter une cuvre d'art, n'était connu généralement que par la version publiée, en 1943, dans Amérique française: on ne pourra plus désormais faire abstraction des différents états de textes qui ont précédé la version connue, principalement ceux qui sont présentés ici sous les intitulés Au printemps dernier et Ce destin, fatalement, s'accomplira.

Le professeur Jean Fisette prend la relève de Bourassa pour la présentation de la troisième partie du volume Écrits I, la plus lourde des cinq puisqu'elle compte à elle seule huit présentations de textes - dont Refus global — tous publiés durant les années 1947-1948. Au cours de ces années prolifiques, le ton des écrits de Borduas change. Celui de l'éditeur de ces textes également qui est amené à nous suggérer, face aux changements de ton (ou plutôt de paradigme) des principaux textes présentés, que Borduas - en toute inconscience? - fonctionne dans la Transformation continuelle suivant le type logique analogique, alors que dans Refus global, il opterait plutôt pour le type logique digital de l'exclusif (à noter que Fisette renvoie complaisamment les non-initiés, en note infrapaginale, pour une présentation sommaire de ces notions techniques, aux ouvrages de Watzlawick et de Wilden...).

Le premier texte présenté dans ce chapitre s'intitule le Retour. Il s'agit d'un manuscrit inédit, non daté, mais qui pourrait être de 1947 et constituerait, en raison des reflexions personnelles qu'y glisse Borduas la matrice des grands textes de Borduas (p. 257). Le manuscrit suivant, Indiscrétions, également inédit et non daté, aurait été rédigé au cours de l'hiver 1947-1948. Dans cet écrit, Borduas trace à grands traits, et non sans humour, le portrait de sept des membres du groupe avec lesquels il travaille et qui ne seront autres que les principaux signataires du Refus global: Barbeau, Cormier, Claude et Pierre Gauvreau, Leduc, Mousseau et Riopelle. Vient ensuite la Transformation continuelle, un texte important, publié partiellement, en 1977, par F.-M. Gagnon et considéré par Fisette comme la synthèse probablement la plus achevée des thèmes typiquement borduasiens (p. 273), même si le traitement de ces thèmes differre fondamentalement de celui qu'on retrouve dans Refus global. Suivent, un très court texte, Parlons un peu peinture, à la défense de l'automatisme, et un autre plus long, Commentaires sur des mots courants, sorte de glossaire et d'essai pédagogique propre à éclairer - semble-t-il - les questions les plus souvent posées lors de débats publics (p. 296).

Puis apparaît Refus global, un moment douloureux, mais une nécessité historique (p. 324), l'aboutissement autant de la pensée personnelle de Borduas que des discussions qui se tenaient dans son groupe (p. 315). Dans l'introduction générale de ce chapitre comme dans la présentation propre à ce texte, J. Fisette donne bonne part aux ambiguittés et aux paradoxes du Borduas de ce manifeste: Borduas qui se méfiait des intellectuels (p. 246), Borduas qui réagissait comme un artiste (p. 247), Borduas à la pensée fluctuante (p. 244), Borduas promoteur de 
l'anarchie resplendissarste (p. 248), mais il résous le tout et principalement le rapport entre le Borduas peintre et le Bordusas ecrivain par cette phase intense de «paradoxe existentiel» que l'homme Borduas rraverse alors. Entendre par là que ce paradoxe existentiel se définit précisément (d'après Watzlawick) comme le produit d'une contradiction entre deux niveaux logiques différents, soit l'analogique (ici l'art, la peinture comme produit du geste spontané) et le digital (ici les lois et conditions régissant cette forme expressive dite spontanée); enfin ce paradoxe trouve (ou non) sa solution da troisieme niveau logique, celui de la métacommunication (ici, le discours tenu sur la peinture). Dans cette perspective, on verra la raison d'être de ces écrits de Borduas sur l'automatisme (p. 250). J. Fisette nous présente, pour compléter ce chapiore, deux courts textes de Borduas, dont l'un, le Surriéslisme ế [nous, représente la première version,

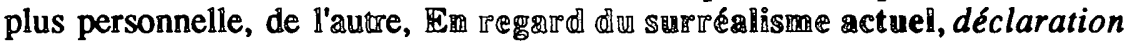
publique d'autonomie (p. 352) par rapport anx surrealistes européens.

La quatrième partie d'tícriôs II est consacrée exclusivement à Projections libérantes. Gilles Lapointe fait d'abord état de l'imposant travail d'édition que requérait ce texte aux nombreuses ébauches, dont certaines erronées, et que Borduas considérait comme le «pecit bouquin» à faire imprimer absolument. Cet ouvrage, qui rapporte surtout les grandes étapes de la carrière de Borduas représentait d'abord et avant coust une libération personnelle (p. 384). Mis en librairie en juillet 1949, il fut source de déception pour Borduas. Pour le public, Borduas n'était plus d'actualité. Bilan du libraire, trois ans après: 192 exemplaires vendus. Il faudra attendre la publication de ce texte par 12 Barre dit jour, en 1969, pour que s'en emparent les criquques qui, depuis, y voient soit un manifeste, soit un pamphlet autobiographique, soit un plaidoyer. G. Lapointe préfere pour sa part le terme «apologie».

André-G. Bourassa revient darns la cinquième et dernière partie du volume pour nous faire connaitre les derniers textes de Borduas, parus entre 1949 et 1958. Dix textes de quelques pages chacun seulement, en forme d'articles ou de lettres 5 .

Telle est, globalement, la somme documentaire que nous présente Écrits $\mathbb{R}$. Un ouvrage de très grande qualité6.

Faut-il conclure pour autaní que cetce remarquable édition critique confère d'autorité un statut littéraire sans conteste ঐ Borduas? Est-il entré par la grande porte dans la «Bibliotheque du Nonveass Monde» pour la qualité intrinseque de ses écrits, pour l'originaliué évidente de ses théories sur l'ant, l'artiste et la société et la beauté formelle de son écriturre, ou plutêt en raison de sa double aura de peintre le plus célèbre du Québec et de chef de la modernitú québécoise?

Le débat est̂ ouvert. Il repose maintenant non seulement sur Refus global, mais sur tous les écrits dont Borduas est le seul signataire. Pour la première fois, grâce à Écrits l, les critiques littéraires ont à la portée de la main la masse imposante d'information et de documentation requise. Tous les écrits de Borduas 
sont là, rassemblés, dans une séquence de publication rigoureusement déterminée, avec textes de base, avant-textes, brouillons et variantes.

La parution des Écrits de Borduas soulèvera sûrement beaucoup d'interrogations, à divers niveaux, et sans doute, cette fois, dans les milieux littéraires d'abord. Aux littéraires, maintenant, de juger de son statut d'écrivain.

1 Paul-Émile Borduas, Écrits I, édition critique par André-G. Bourassa, Jean Fisette et Gilles Lapointe, Montréal, Les Presses de l'Université de Montréal, coll. «Bibliothèque du Nouveau Monde», 1987, $700 \mathrm{p}$.

2 Parmi les inédits qui paraissent dans ce volume, citons notamment dans leur ordre de datation, Perspective (1933), Composition décorative (1937), Enquêtes sur l'artisanat et le tourisme (1938), Inventaires des euvres d'art (1939), Réponse des indépendants à Mallíard (1942), Indiscrétions (1947), le Retour (1947), Protestation d'un groupe de peintres (1948).

3 Ajoutons d'ailleurs qu'André-G. Bourassa, l'initiateur de ce projet d'édition, tient à souligner dans son introduction générale que ces Écrits I doivent beaucoup à certains devanciers de taille, nommément: les professeurs François-Marc Gagnon et Laurier Lacroix, retenus comme consultants, et Pierre Théberge, directeur du Musée des beaux-arts de Montréal. Personne ne peut oublier, en effet, que ces Écrits I paraissent après la monumentale biographie critique de Borduas par F.-M. Gagnon.

4 Ce qui compte ici, ce sont surtout les spicilèges qui sont annexés à ces dossiers et qui contiennent au total quelque sept cents photographies annotées. Les photos de Tessier sont bien connues; celles de Borduas, pas du tout. Ecrits I reproduit ici, pour la toute première fois, quarante des photos prises par Borduas. Inutile de dire qu'elles traduisent un regard inconnu de Borduas sur les paysages et «natures mortes» (tapis crocheté, vadrouille, dévidoir, enseigne...) du Québec.

5 Les Expositions itinérantes (en réponse à un article du critique David Mawr du Daily Star); Communication intime à mes chers amis (dont le contenu porte surtout sur le débat entre art et engagement, sur les rapports entre automatistes et collectivité, et à la fin, sur les ruptures personnelles de l'auteur); Quelques Pensées sur l'euvre d'amour et de rêve de $M$. Ozias Leduc (1953) et Après tant de siècles de silences (1954) (deux impressionnants témoignages de Borduas sur son maître Ozias Leduc); $\mathbf{O b}$ jectivation ultime ou délirante (1955; sur les destinées de l'art non figuratif); Propos d'atelier (1956; lettre sur l'actualité de l'art à la demande de Noël Lajoie du Devoir); Questions et réponses (1956; suite à une enquête de Jean-René Ostiguy qui préparait alors l'exposition "Canadian Abstract Paintings» pour la Galerie nationale du Canada); la Chambre ouverte (calligramme et lettre accompagnant une toile destinée à deux jeunes amis de France, Lou et Michel Camus); Approximations (1958; réponses à un questionnaire de la revue Situations, dix ans après Refus global); Petite Pierre angulaire posée dans la tourbe de mes vieux préjugés (1958; lettre adressée à Claude Gauvreau et dont le contenu passe des confidences personnelles aux grandes questions nationalistes). Jusqu'à nouvel ordre, de conclure Bourassa, nous considérons que le dernier texte de Borduas est 


\section{CHRONIQUES 483}

Petite Pierre angulaire (p. 498).

6 Il est dommage, par ailleurs, que la présentation typographique de ces textes ne permette pas au lecteur de distinguer plus facilement entre les introductions des auteurs et les textes de Borduas eux-mêmes. D'autant plus que la table des matières comporte des erreurs gênantes: Fusaln n'est pas à la page 151, mais à la page 165, Au printemps dernier est à la page 167, et non 155 . Autres erreurs relevées au passage: le nom du peintre d'origine italienne Guido Nincheri n'est pas Ninchri (p. 24), ni Ninchéri (p. 27), mais bien Nincheri. L'architecte Georges Bouillon n'est pas né à Ottawa, mais à Saint-Germain de Rimouski et il est décédé à l'âge de 91 ans, non de 89 (p. 549). 